\title{
Sécheresse climatique au Maroc durant les dernières décennies
}

\section{Climatic drought in Morocco during the last decades}

\author{
L. Stour(1), A. Agoumi(2) \\ (1) Professeur à la faculté des sciences et techniques de Mohammedia, BP 146, Mohammedia, Maroc \\ e-mail : stourl@gmail.com \\ (2) Professeur à l'école Hassania des travaux publics, BP 8108 Oasis, Casablanca, Maroc \\ e-mail : agoumi.ali@gmail.com
}

Résumé - La sécheresse a toujours été présente dans l'histoire du Maroc, elle s'est imposée avec force ces dernières décennies en tant qu'élément structurel du climat du pays. Le Maroc vit actuellement l'épisode sec le plus long de son histoire contemporaine, caractérisé par une diminution des précipitations et une tendance nette à la hausse des températures. La caractérisation de la sécheresse climatique au cours de la période 19612004 a montré une augmentation significative de la fréquence des sécheresses, de leur sévérité et de leur champ de couverture spatiale. Cette dynamique de sécheresse s'accompagne d'un réchauffement important lié au changement climatique planétaire, qui rend ces années sèches de plus en plus difficiles pour différents secteurs socio-économiques du pays.

Mots clés - sécheresse, réchauffement, précipitation, température, Maroc

Abstract - Drought was always present in Morocco's history. Its importance as a structural element of the country's climate increased these last decades. Morocco lives the longest dry episode of its contemporary history, characterized by a reduction of the precipitations and a tendency to the rise in temperatures. The characterization of the climatic drought during the period 1961-2004 showed an increase in the droughts frequency, their severity and their spatial distribution. This drought dynamics is associated to an important warming up related to the global climate change that makes these dry episodes more and more difficult for different socioeconomic sectors.

Key words - drought, warming, precipitation, temperature, Morocco

\section{INTRODUCTION}

La sécheresse est l'une des catastrophes naturelles qui ont marqué profondément la vie des populations à travers les âges. Elle n'a pas de définition universelle, il y a autant de définitions de la sécheresse qu'il y a d'utilisation de l'eau : la sécheresse est en effet un déficit de disponibilité en eau par rapport à une situation 
considérée comme normale pour une période donnée, une région déterminée et des usages spécifiques.

On distingue quatre grands types de sécheresse : météorologique, agricole, hydrologique et socioéconomique. Ces types de sécheresse peuvent ne pas se manifester simultanément, mais la sécheresse météorologique reste l'élément moteur des autres. Elle se caractérise par une réduction ou une mauvaise répartition, voir une absence des pluies dans une région donnée pendant une période de temps (Bootsma et al., 1996).

La sécheresse a toujours été présente dans l'histoire du Maroc, des études dendrochronologiques (Stockton, 1988) ont montré que le Maroc a souvent connu dans le passé des périodes d'intenses sécheresses. Ces sécheresses revenaient de façon périodique et amenaient parfois des famines et épidémies. Ainsi, la sécheresse de 1597-1608 a engendré une famine qui a exterminé le tiers de la population (Safi, 1990). Les sécheresses ont été aussi la cause de mouvements migratoires importants dans la région.

La sécheresse reste une composante forte du climat contemporain du Maroc : Une étude récente (Ministère de l'équipement et al., 1997) a montré que depuis 1896 jusqu'à 1996 le Maroc a connu onze périodes de sécheresses généralisées dont l'intensité a été modérée à forte et d'autres moins généralisées mais assez fortement ressenties. Le Maroc vit actuellement l'épisode sec le plus long de son histoire contemporaine (DMN, 1995). Durant les cinquante dernières années, on est passé d'une sécheresse tout les dix ans les années 50-60 à deux à trois sécheresses par décennie (Agoumi \& Debbarh, 2005).

En plus du caractère structurel de la récurrence des sécheresses, ces dernières deviennent de plus en plus sévères, comme en a témoigné la dernière sécheresse de 1994-95 qui a paralysé l'économie du pays, en particulier le secteur agricole qui contribue en moyenne par $17 \%$ au produit intérieur brut.

Si la sécheresse prend une dimension importante au Maroc c'est qu'elle conditionne un secteur clé de l'économie nationale : l'agriculture dont près de $85 \%$ est pluviale, soumise directement aux aléas climatiques.

Les impacts de la sécheresse sont non structurels, complexes et difficiles à quantifier, contrairement aux impacts des autres risques naturels (Wilhite \& Svoboda, 2000). La complexité de ces impacts est due à la diversité des secteurs qui utilisent l'eau pour la production des biens et services. Ils peuvent être classés en trois catégories : économique, social et environnemental.

Différentes études et recherches ont été menées à ce jour au Maroc et ont concerné les sécheresses agricoles et hydrologiques. Ces études ont concerné les années précédant 1995. Ainsi, le comportement de ce phénomène durant les dix dernières années n'a fait l'objet d'aucune recherche. Cette décennie est pourtant singulière. Cela a été mis en exergue dans le quatrième rapport de groupe d'experts intergouvernemental sur l'évolution du climat (GIEC) qui caractérise l'évolution des changements climatiques au niveau planétaire (GIEC, 2007).

La présente étude a cherché à combler cette lacune. Notre objectif est de 
caractériser la sécheresse météorologique au Maroc sur la période 19612004, avec un intérêt particulier pour la période 1993-2004, période durant laquelle, les travaux de GIEC ont montré que le climat planétaire a connu un réchauffement important accompagné d'événements extrêmes aigues dont les sécheresses et les inondations.

Nous nous sommes intéressés à l'évolution des précipitations $(P)$, des températures ( $T$ ) et de l'évapotranspiration (ETP) à travers différents indices et ce à partir de données relevées par la direction de la météorologie nationale (Tilioua \& Mechouary, 2006) dans sept stations représentatives de la majorité des zones climatiques du pays (Ministère de l'équipement et al., 1997) : Tanger, Oujda, Rabat, Casablanca, Marrakech, Ifrane, et Agadir.

\section{APPROCHE ADOPTÉE}

Les travaux de recherche récents les plus marquants ont étudié la sécheresse au Maroc sur la base de ses effets. Ainsi, Barakat \& Handoufe (1998) se sont basés sur une approche fondée sur la production agricole pour caractériser la sécheresse, et ont défini un indicateur de ce phénomène sur la base du cumul de déficit pluviométrique du milieu de la campagne agricole. Yacoubi et al. (1998) se sont intéressés à la typologie de la sécheresse agricole céréalière et ont défini une méthode d'alerte précoce à la sécheresse.

Notre approche ici est différente. Elle est basée sur une caractérisation de la sécheresse en utilisant des indices employés dans différentes régions du monde. Ces indices permettent de déterminer le seuil indiquant la sécheresse à différentes échelles de temps et de définir les classes d'appartenance à cet événement en fonction de sa sévérité.

Pour la période 1961-2004, l'analyse faite a intégré les étapes suivantes:

- analyse statistique des séries de précipitations et de températures relevées dans les différentes stations durant cette période;

- évaluation à partir de ces données de l'ETP et analyse de son comportement;

- estimation des indices de sécheresse météorologique et analyse de la dynamique climatique au Maroc.

\section{INDICES DE SÉCHERESSE MÉTÉOROLOGIQUE UTILISÉS}

Pour caractériser et identifier la sécheresse climatique, plusieurs méthodes et indices ont été utilisés à différentes échelles de temps : mensuelle, saisonnière et annuelle.

Ils permettent de déterminer le seuil de la sécheresse climatique, d'établir une classification selon la sévérité de l'événement et selon sa position et d'identifier la probabilité d'occurrence de différentes classes de sévérité.

\subsection{Méthode d'intervalle de confiance (IC)}

Cette méthode n'est applicable que pour les distributions qui suivent un modèle normal. Pour un niveau de confiance $(1-\alpha)$ de $95 \%$, les 
Tableau I. Méthode d'intervalle de confiance.

Table I. Confidence interval method.

\begin{tabular}{lll}
\hline Marge d'erreur $(\mathrm{E})$ & Limite supérieure (Ls) & Limite inférieure (Li) \\
\hline $\mathrm{T} 1-\alpha / 2 ; \mathrm{n}-1 *\left[\mathrm{~s} / \mathrm{n}^{1 / 2}\right]$ & $\mathrm{M}+\mathrm{T} 1-\alpha / 2 ; \mathrm{n}-1 *\left[\mathrm{~s} / \mathrm{n}^{1 / 2}\right]$ & $\mathrm{M}-\mathrm{T} 1-\alpha / 2 ; \mathrm{n}-1 *\left[\mathrm{~s} / \mathrm{n}^{1 / 2}\right]$ \\
\hline $\mathrm{M}$ & $:$ Moyenne estimée \\
$\mathrm{T} 1-\alpha / 2 ; \mathrm{n}-1$ & $:$ Valeur de la loi T STUDENT de $1-\alpha / 2$ à $\mathrm{n}-1$ degré de liberté \\
$\mathrm{s} / \mathrm{n}^{1 / 2}$ & $:$ Erreur type \\
$\mathrm{s}$ & $:$ Écart type de l'échantillon \\
$\mathrm{n}$ & $:$ Taille de l'échantillon
\end{tabular}

bornes inférieures et supérieures de l'intervalle de confiance sont calculées comme suit (Tab. I) :

En effet, on a considéré la limite inférieure de l'intervalle de confiance comme seuil de sécheresse. C'est-àdire que toute période qui a enregistré une quantité de précipitation inférieure à cette limite est qualifiée climatiquement sèche. Également, toute période qui a connu une précipitation comprise entre la borne inférieure et la borne supérieure est considérée normale. Alors qu'on dit qu'une période est humide lorsque la pluviométrie dépasse la limite supérieure de l'intervalle de confiance.

En se basant sur les seuils qui indiquent la période sèche, normale et humide, on a déterminé la probabilité d'occurrence de chacun de ces trois événements pour chaque période de temps.

\subsection{Rapport à la normale des précipitations (RN)}

II est exprimé mathématiquement comme suit :

$$
\mathrm{RN}(\%)=(\mathrm{Pi} / \mathrm{Pn}) \times 100
$$

Pi est la précipitation de l'année i pour la période 1961-2004 et $\mathrm{Pn}$ est la précipitation normale pour la même période de temps.

Une année sèche est une année dont la pluviométrie se situe audessous de la normale; c'est-à-dire lorsque le RN est inférieur à $100 \%$ (Rognon, 1997).

\subsection{Indice de l'écart à la normale (En)}

Cet indice nommé aussi indice de déficit pluviométrique, permet de visualiser et de déterminer le nombre des années déficitaires et leur succession. Elle s'exprime par :

$$
\mathrm{En}(\%)=(\mathrm{Pi}-\mathrm{Pn}) / \mathrm{Pn} \times 100
$$

Une année est dite sèche lorsque cet indice est négatif et humide quand il est positif. Le cumul de l'indice de l'écart en pourcentage à la normale des années successives permet de dégager les grandes tendances en faisant abstraction des faibles fluctuations d'une année à l'autre. Quand la somme des indices croit, il s'agit d'une tendance humide. 
Tableau II. Sévérité de la sécheresse selon la méthode de nombre d'écart type.

Table II. Drought Severity according to standard deviation number method.

\begin{tabular}{ll}
\hline Classes & Seuils des classes de sévérité \\
\hline Extrêmement humide & $\mathrm{Pi}>\mathrm{Pm}+2 \sigma$ \\
Sévèrement humide & $\mathrm{Pm}+\sigma<\mathrm{Pi}<\mathrm{Pm}+2 \sigma$ \\
Modérément humide & $\mathrm{Ls}<\mathrm{Pi}<\mathrm{Pm}+\sigma$ \\
Normale & $\mathrm{Li}<\mathrm{Pi}<\mathrm{Ls}$ \\
Sécheresse modérée & $\mathrm{Pm}-\sigma<\mathrm{Pi}<\mathrm{Li}$ \\
Sécheresse sévère & $\mathrm{Pm}-2 \sigma<\mathrm{Pi}<\mathrm{Pm}-\sigma$ \\
Sécheresse extrême & $\mathrm{Pi}<\mathrm{Pm}-2 \sigma$ \\
\hline
\end{tabular}

La tendance est sèche, dans le cas contraire.

\subsection{Indice de nombre d'écart type}

Cet indice se base sur la comparaison de la pluviométrie actuelle au nombre d'écart type comme indiqué dans le tableau II (Aghrab, 2003).

\subsection{Indice standardisé de précipitation}

L'indice standardisé de précipitation «SPI » (Standardised precipitation index) a été développé en vue de caractériser les déficits de précipitation pour une période donnée (Mckee et al., 1993). Il prend en considération l'importance du temps dans l'analyse de la disponibilité des ressources en eau. En effet, la période de temps sur la quelle le déficit des précipitations est accumulé devient extrêmement importante pour séparer entre les différents types de sécheresses (Mckee et al., 1995). Il peut être calculé à différentes échelles de temps $(1,3$, $6,12,24$ et 48 mois) afin de refléter l'impact de la sécheresse sur les différentes ressources en eau (Hayes et al., 1999).

La formule mathématique de SPI est la suivante :

$$
\mathrm{SPI}=(\mathrm{pi}-\mathrm{pm}) / \sigma
$$

$\mathrm{Pi}$ : Précipitation de l'année i

$\mathrm{Pm}$ : Précipitation moyenne

$\sigma$ : Déviation standard ou écart type

Les classes de sévérité sont définies arbitrairement (Mckee et al., 1993). En utilisant les seuils de sévérité établis par la méthode de nombre d'écart type, (Aghrab, 2003) a établi une nouvelle classification de SPI qu'il a nommé SPI corrigé ou SPIc. Cette nouvelle classification est présentée dans le tableau III. 
Tableau III. Classification de la sévérité de la sécheresse selon SPIc (Aghrab, 2003) en comparaison avec celle proposée par (Mckee et al., 1993).

Table III. Drought severity classification according to SPIc (Aghrab, 2003) in comparison with (Mckee et al., 1993).

\begin{tabular}{lccc}
\hline Classes & $\begin{array}{c}\text { Classification } \\
\text { Selon SPI }\end{array}$ & $\begin{array}{c}\text { Seuils des classes de } \\
\text { Sévérité }\end{array}$ & $\begin{array}{c}\text { Classification } \\
\text { selon SPIc }\end{array}$ \\
\hline Extrêmement humide & Plus de 2,0 & $\mathrm{Pi}>\mathrm{Pm}+2 \sigma$ & Plus de 2 \\
Sévèrement humide & $1,5 \mathrm{à} 1,99$ & $\mathrm{Pm}+\sigma<\mathrm{Pi}<\mathrm{Pm}+2 \sigma$ & 1 à 1,99 \\
Modérément humide & 1,0 à 1,49 & $\mathrm{Ls}<\mathrm{Pi}<\mathrm{Pm}+\sigma$ & 0,31 à 0,99 \\
Normale & $-0,99$ à 0,99 & $\mathrm{Li}<\mathrm{Pi}<\mathrm{Ls}$ & $-0,30$ à 0,30 \\
Modérément sèche & $-1,0$ à $-1,49$ & $\mathrm{Pm}-\sigma<\mathrm{Pi}<\mathrm{Li}$ & $-0,31$ à $-0,99$ \\
Sévèrement sèche & $-1,5$ à $-1,99$ & $\mathrm{Pm}-2 \sigma<\mathrm{Pi}<\mathrm{Pm}-\sigma$ & -1 à $-1,99$ \\
Extrêmement sèche & Moins de $-2,0$ & $\mathrm{Pi}<\mathrm{Pm}-2 \sigma$ & Moins de -2 \\
\hline
\end{tabular}

\section{RÉSULTATS ET ANALYSE}

\section{1 Évolution des précipitations}

Les caractéristiques statistiques de base des séries pluviométriques des sept villes étudiées sont récapitulées dans le tableau IV. L'étude de la variabilité climatique interannuelle montre que la pluviométrie annuelle se caractérise par une forte fluctuation surtout à Agadir ou le coefficient de variation atteint $50 \%$ contre $30 \%$ à $40 \%$ dans les autres villes. L'écart entre le minimum et le maximum est très important.
La précipitation annuelle a connu une tendance nette à la baisse au niveau des sept villes étudiées (Fig. 1), en particulier au cours des douze dernières années. La moyenne des précipitations au cours de cette période est généralement inférieure à celle de la période 1961-2004 (Fig. 2).

L'analyse de l'évolution de l'écart cumulé des précipitations par rapport à la normale (Fig. 3) a mis en exergue deux périodes : une première période pluvieuse et normale de 1961 à 1972 (cumul des écarts croît principalement), puis une période sèche très peu pluvieuse entre 1972

Tableau IV. Caractéristiques statistiques des précipitations annuelles pour la période 1961-2004.

Table IV. Statistical characteristics of the yearly precipitations for the period 1961-2004.

\begin{tabular}{lrcrrrrr}
\hline Caractéristiques & Agadir & Casablanca & Ifrane & Marrakech & Oujda & Rabat & Tanger \\
\hline Moyenne (mm) & 255,05 & 418,65 & 968,95 & 236,63 & 307,85 & 539,25 & 736,13 \\
Médiane (mm) & 228,05 & 408,65 & 890,15 & 237,45 & 297,10 & 508,75 & 714,00 \\
Minimum (mm) & 45,70 & 148,50 & 605,00 & 80,90 & 109,00 & 249,60 & 412,00 \\
Maximum (mm) & 670,00 & 991,60 & 1865,70 & 427,00 & 555,30 & 1163,60 & 1699,40 \\
Écart-type (mm) & 128,85 & 154,61 & 305,23 & 86,72 & 100,38 & 181,01 & 237,63 \\
Coef.variation (\%) & 50,52 & 36,93 & 31,50 & 36,65 & 32,61 & 33,57 & 32,28 \\
Coef.asymétrie (\%) & 143,90 & 153,59 & 147,96 & 20,87 & 49,10 & 100,16 & 182,50 \\
Etendu (mm) & 624,30 & 843,10 & 1260,70 & 346,10 & 446,30 & 914,00 & 1287,40 \\
\hline
\end{tabular}



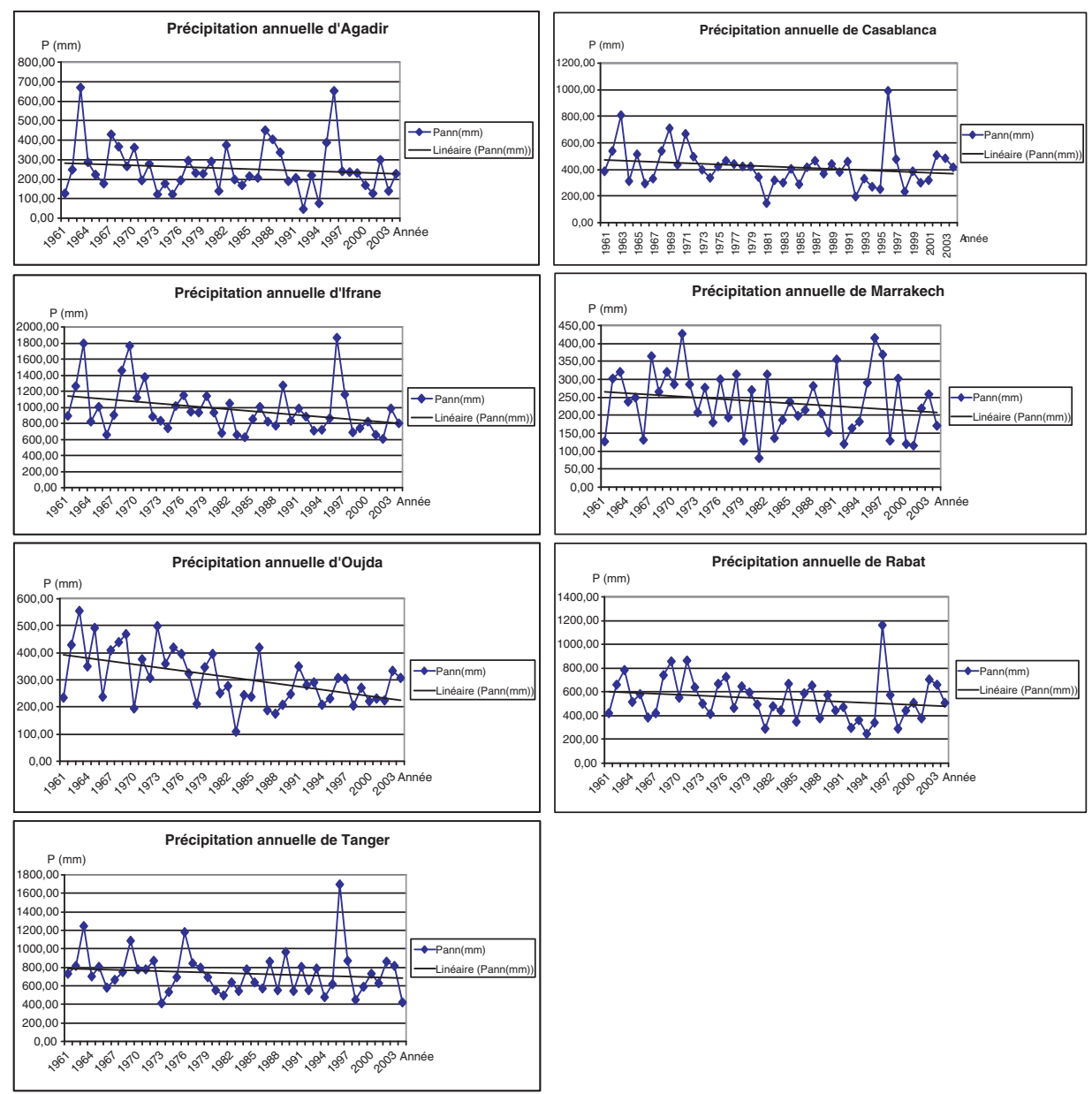

Fig. 1. Évolution des précipitations annuelles dans les sept villes au cours de la période 1961-2004.

Fig. 1. Yearly precipitations in the seven cities during the period 1961-2004.

et 2004 (cumul des écarts décroît), avec deux épisodes secs, longs et critiques : $1972-1995$ et $1997-2004$. Cette seconde période (1972-2004) est entrecoupée de quelques courtes périodes à tendance humide, surtout l'année 1996 qui était extrêmement humide dans la majorité du territoire nationale.

\section{2 Évolution de la température et de l'évapotranspiration potentielle}

Les températures moyennes annuelles se caractérisent cette fois-ci par une faible variation d'une année à l'autre, le coefficient de variation fluctue entre 3 et $6 \%$ (Tab. V). 


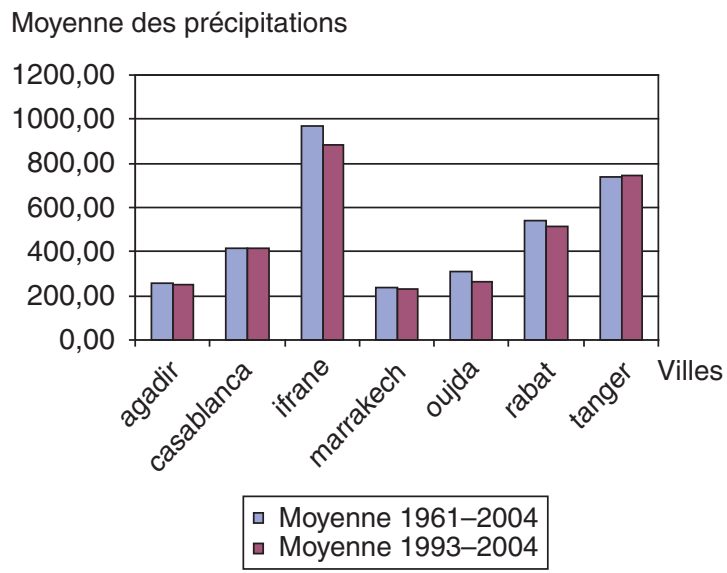

Fig. 2. Moyennes de précipitations de deux périodes 1961-2004 et 1993-2004 pour les sept villes.

Fig. 2. Precipitations averages for two periods 1961-2004 and 1993-2004 in the seven cities.

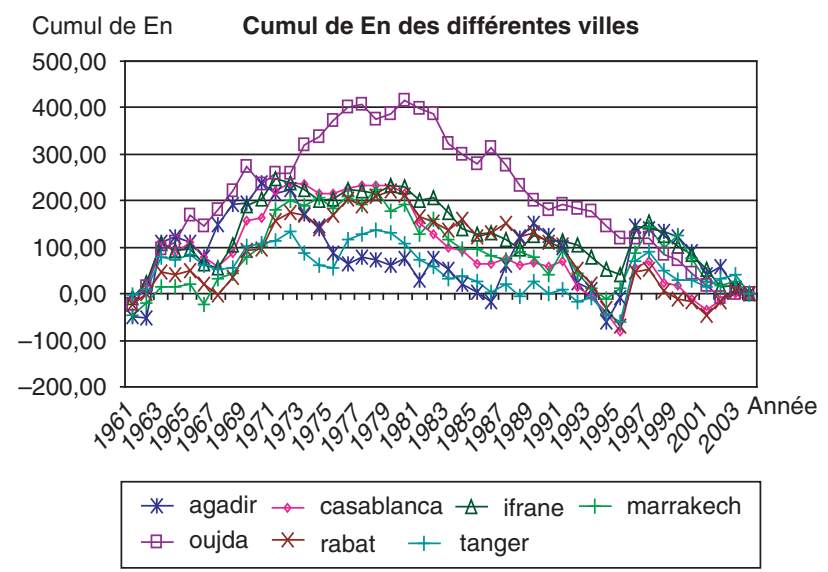

Fig. 3. Évolution de l'écart cumulé des précipitations par rapport à la normale dans les sept villes. Fig. 3. Precipitations accumulated variation in relation to the normal in the seven cities.

L'analyse de l'évolution des températures au cours de la période 19612004 (Figs. 4 et 5) indique l'existence de deux périodes significatives du point de vue thermique :

- La période 1961-1972 a connu un refroidissement progressif du climat de l'ordre de $1^{\circ} \mathrm{C}$ en moyenne annuelle;
- La période 1972-2004 a connu un réchauffement important, où des records absolus de températures ont été battus (Agoumi \& Debbarh, 2005).

Au-delà du refroidissement des années 70 , la température moyenne a connu une tendance nette à la hausse au niveau des sept villes : plus de $1^{\circ} \mathrm{C}$ 
Tableau V. Caractéristiques statistiques des températures moyennes annuelles pour la période 19612004.

Table V. Statistical characteristics of the yearly average temperatures for the period 1961-2004.

\begin{tabular}{lrrrrrrr}
\hline Caractéristiques & Agadir & Casablanca & Ifrane & Marrakech & Oujda & Rabat & Tanger \\
\hline Moyenne $\left({ }^{\circ} \mathbf{C}\right)$ & 18,27 & 17,28 & 11,36 & 19,46 & 16,56 & 17,04 & 17,50 \\
Médiane $\left({ }^{\circ} \mathbf{C}\right)$ & 18,21 & 17,38 & 11,27 & 19,49 & 16,45 & 17,02 & 17,51 \\
Minimum ( $\left.{ }^{\circ} \mathbf{C}\right)$ & 16,55 & 15,53 & 9,41 & 17,63 & 15,22 & 15,83 & 16,13 \\
Maximum ( $\left.{ }^{\circ} \mathbf{C}\right)$ & 19,41 & 18,65 & 12,79 & 20,55 & 18,04 & 18,18 & 18,45 \\
Écart-type ( $\left.{ }^{\circ} \mathbf{C}\right)$ & 0,65 & 0,67 & 0,75 & 0,65 & 0,73 & 0,51 & 0,51 \\
Coeff. variation (\%) & 3,55 & 3,90 & 6,64 & 3,34 & 4,40 & 2,99 & 2,90 \\
Coeff. asymétrie (\%) & $-11,16$ & $-26,55$ & $-14,70$ & $-56,81$ & $-3,77$ & 19,88 & $-29,71$ \\
Etendu ( $\left.{ }^{\circ} \mathbf{C}\right)$ & 2,86 & 3,12 & 3,38 & 2,91 & 2,83 & 2,35 & 2,32 \\
\hline
\end{tabular}

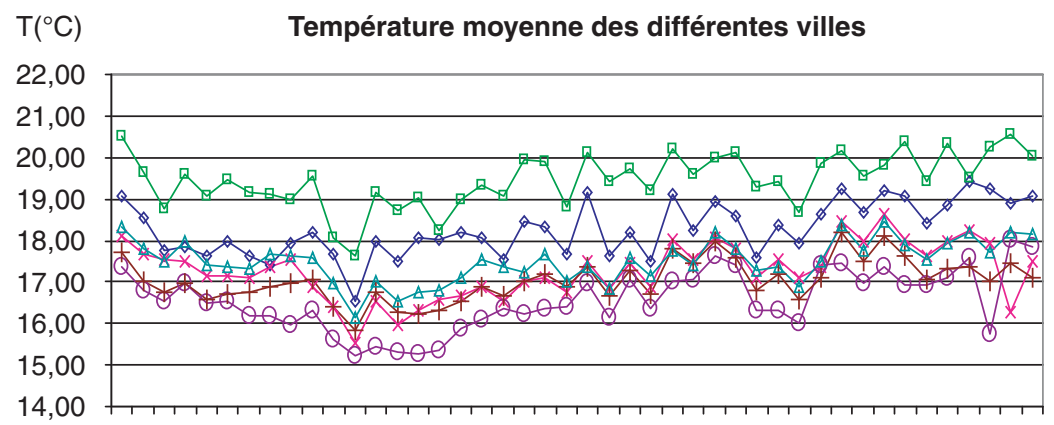

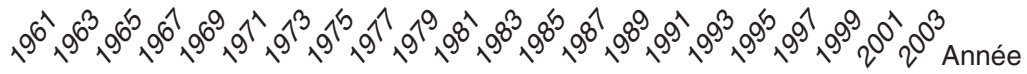

\begin{tabular}{lllll}
\hline$\rightarrow$ Tagadir & $*$ Tcasablanca & $\rightarrow$ & Tmarrakech \\
- & Toujda $\rightarrow$ Trabat & $\rightarrow$ & Ttanger \\
\hline
\end{tabular}

Fig. 4. Évolution des températures moyennes annuelles au cours de la période 1961-2004.

Fig. 4. Yearly average temperatures during the period 1961-2004.

de réchauffement entre 1972 et 2004 (32 ans).

Le réchauffement a été fort au cours des douze dernières années. La moyenne des températures au cours de cette période est supérieure à celle de la période 1961-2004, elle la dépasse de plus de $0,6^{\circ} \mathrm{C}$ pour la ville d'Agadir (Fig. 6). Cette tendance est conforme au réchauffement global planétaire enregistré durant les cinquante dernières années avec l'accélération de ce processus durant la période 1993-2005 qui figure au palmarès des épisodes les plus chauds (GIEC, 2007). 


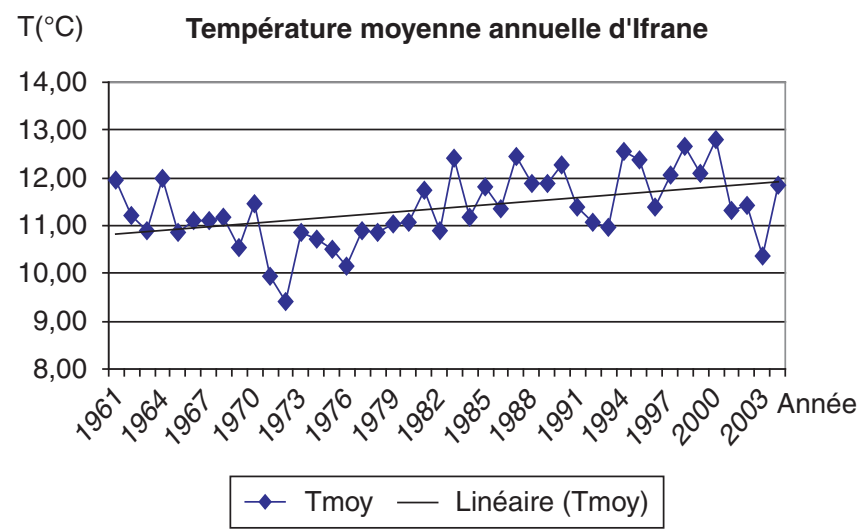

Fig. 5. Températures moyennes annuelles d'Ifrane au cours de la période 1961-2004.

Fig. 5. Yearly average temperatures in Ifrane during the period 1961-2004.

L'évapotranspiration potentielle ${ }^{(1)}$ fortement influencée par la température de l'air a connu logiquement au cours de la période 1961-2004 une tendance à la hausse (Figs. 7 et 8 ). Cette augmentation de l'ETP associée à la diminution relevée des précipitations a eu certainement un effet néfaste sur les potentialités en eau du royaume (Agoumi \& Debbarh, 2005).

\subsection{Caractérisation de la sécheresse météorologique}

La méthode d'intervalle de confiance appliquée à notre base de données a permis d'estimer les seuils qui

(1) Pour l'estimation de l'ETP, nous avons utilisé la formule de Blanney-criddle : ETP = P $(0,46$ Tmoy + 8); ETP : Evapotranspiration potentielle journalière ( $\mathrm{mm} /$ jour) ; Tmoy : Température moyenne journalière $\left({ }^{\circ} \mathrm{C}\right) ; \mathrm{P}$ : Pourcentage journalier moyen des heures annuelles de lumière diurne, il varie en fonction de la latitude de la région et du mois considéré. indiquent la sécheresse climatique annuelle dans les sept villes (Tab. VI). Ces seuils calculés oscillent entre $876 \mathrm{~mm}$ à Ifrane et $210 \mathrm{~mm}$ à Marrakech. Ils ont permis de déterminer les seuils relatifs aux indices de l'écart à la normale et du rapport à la normale (Tab. VI).

L'analyse de ces résultats montre la grande vulnérabilité du versant occidental du moyen-atlas (Ifrane avec un seuil de $876 \mathrm{~mm}$ ) et de l'extrême nordouest du Maroc (Tanger avec un seuil de $663 \mathrm{~mm}$ ) vis-à-vis de la sécheresse.

En utilisant les seuils de sécheresse calculés ci-dessus, on a pu déterminer pour chacune des stations étudiées les fréquences des années sèches, normales et humides (Tab. VII).

Les années sèches représentent entre $46 \%$ et $39 \%$ respectivement à Oujda et Casablanca. Les sécheresses les plus persistantes sont survenues au cours de deux dernières décennies, elles sont formées de trois, quatre et cinq années 


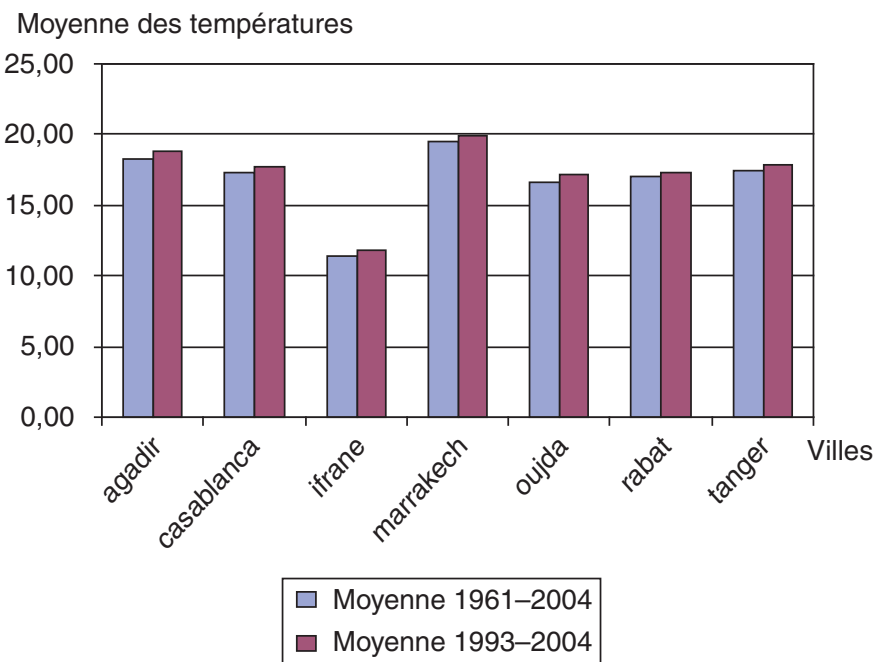

Fig. 6. Moyenne des températures annuelles de deux périodes 1961-2004 et 1993-2004.

Fig. 6. Average of yearly temperatures of two periods 1961-2004 and 1993-2004.

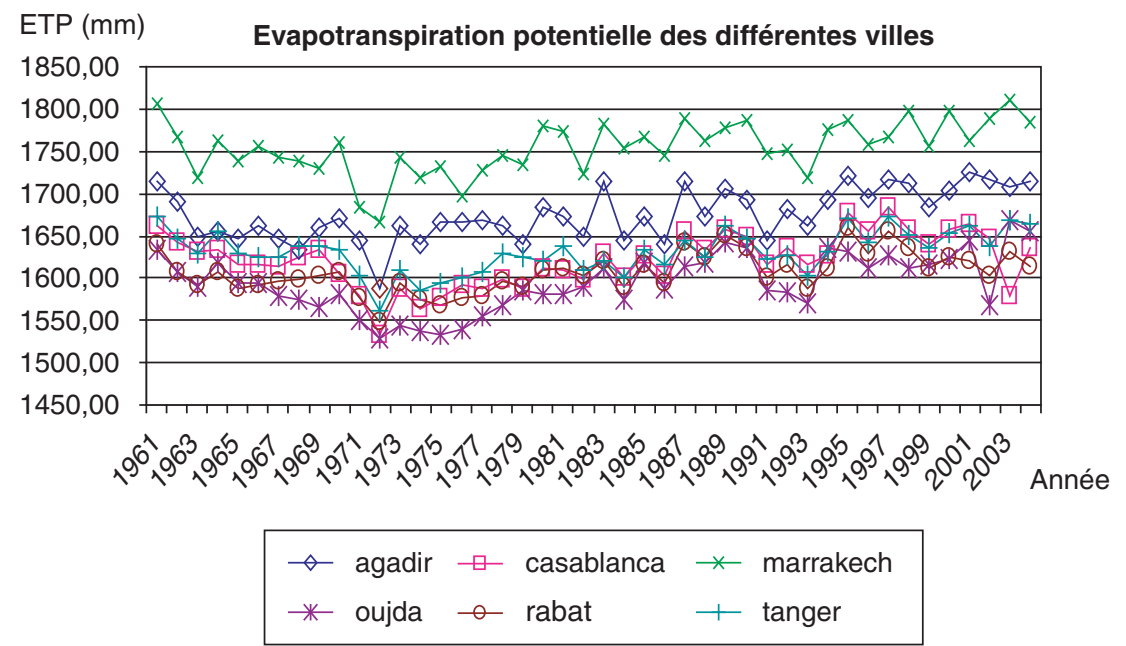

Fig. 7. Évolution de l'évapotranspiration potentielle au cours de la période 1961-2004.

Fig. 7. Potential evapotranspiration during the period 1961-2004. 


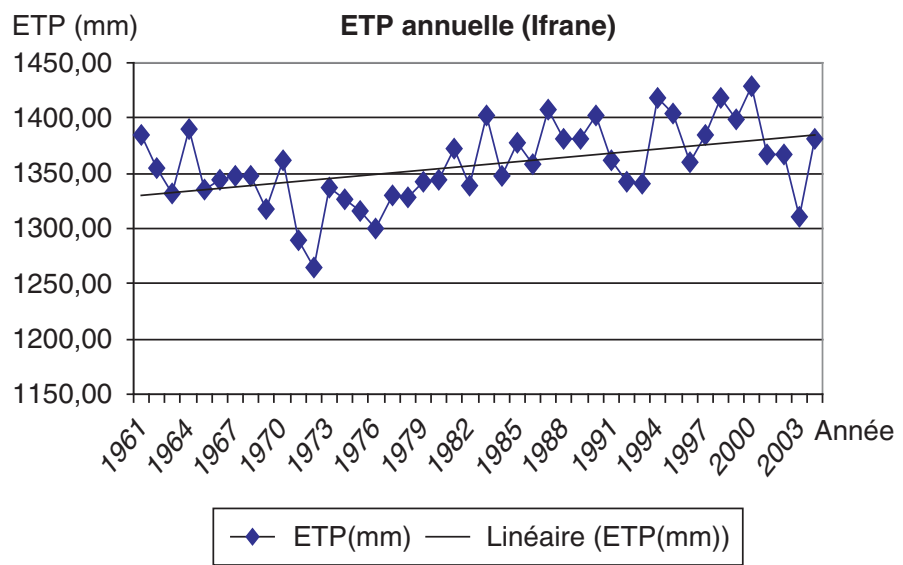

Fig. 8. Évolution de l'évapotranspiration potentielle de la ville d'Ifrane au cours de la période 19612004.

Fig. 8. Potential evapotranspiration in Ifrane during the period 1961-2004.

Tableau VI. Seuils de sécheresse correspondants aux différents indices au cours de la période 19612004.

Table VI. Drought limits corresponding to different index during the period 1961-2004.

\begin{tabular}{lccc}
\hline Villes & $\begin{array}{c}\text { Seuil de la sécheresse } \\
(\text { Li de I'IC) }(\mathbf{m m})\end{array}$ & $\begin{array}{c}\text { Seuil de sécheresse de } \\
\text { l'écart à la normale }\end{array}$ & $\begin{array}{c}\text { Seuil de sécheresse du } \\
\text { rapport à la normale }\end{array}$ \\
\hline Agadir & 215,87 & $-15,36 \%$ & $84,64 \%$ \\
Casablanca & 371,62 & $-11,23 \%$ & $88,77 \%$ \\
Ifrane & 876,12 & $-9,58 \%$ & $90,42 \%$ \\
Marrakech & 210,26 & $-11,15 \%$ & $88,85 \%$ \\
Oujda & 277,32 & $-9,92 \%$ & $90,08 \%$ \\
Rabat & 484,19 & $-10,21 \%$ & $89,79 \%$ \\
Tanger & 663,86 & $-9,82 \%$ & $90,18 \%$ \\
\hline
\end{tabular}

Tableau VII. Fréquence des années sèches, normales et humides dans les sept villes au cours de la période 1961-2004.

Table VII. Dry, normal and humid years frequency in the seven cities during the period 1961-2004.

\begin{tabular}{lccc}
\hline Villes & $\begin{array}{c}\text { Fréquence des années } \\
\text { sèches }\end{array}$ & $\begin{array}{c}\text { Fréquence des années } \\
\text { normales }\end{array}$ & $\begin{array}{c}\text { Fréquence des années } \\
\text { humides }\end{array}$ \\
\hline Agadir & $43 \%$ & $32 \%$ & $25 \%$ \\
Casablanca & $39 \%$ & $34 \%$ & $27 \%$ \\
Ifrane & $45 \%$ & $30 \%$ & $25 \%$ \\
Marrakech & $43 \%$ & $14 \%$ & $43 \%$ \\
Oujda & $46 \%$ & $18 \%$ & $36 \%$ \\
Rabat & $43 \%$ & $23 \%$ & $34 \%$ \\
Tanger & $41 \%$ & $32 \%$ & $27 \%$ \\
\hline
\end{tabular}



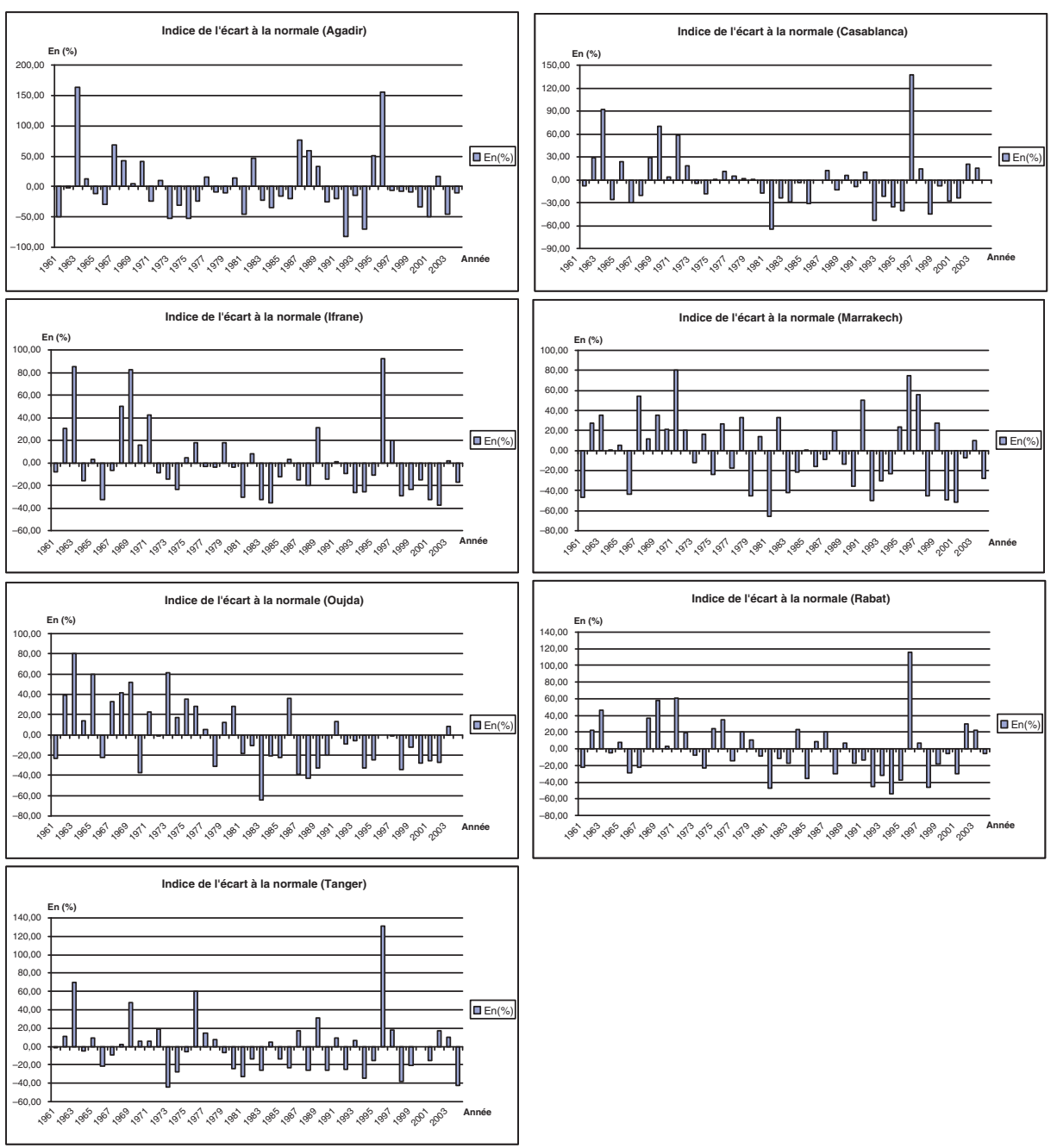

Fig. 9. Écart à la normale au cours de la période 1961-2004 dans les sept villes.

Fig. 9. Variation from the normal during the period 1961-2004 in the seven cities.

sèches consécutives, tandis que les sécheresses des années 60 et 70 ont été souvent isolées (Fig. 9).

L'analyse de différentes sécheresses vécues au niveau de sept villes présentée sur la figure 10 permet de confirmer une fois de plus l'assèchement accru qu'a connu la période 1972-2004:

- avant 1972, les sécheresses étaient peu fréquentes et touchaient rarement l'ensemble des villes; 


\section{Pourcentage des régions touchées par les différentes années de sécheresse à l'échelle nationale}

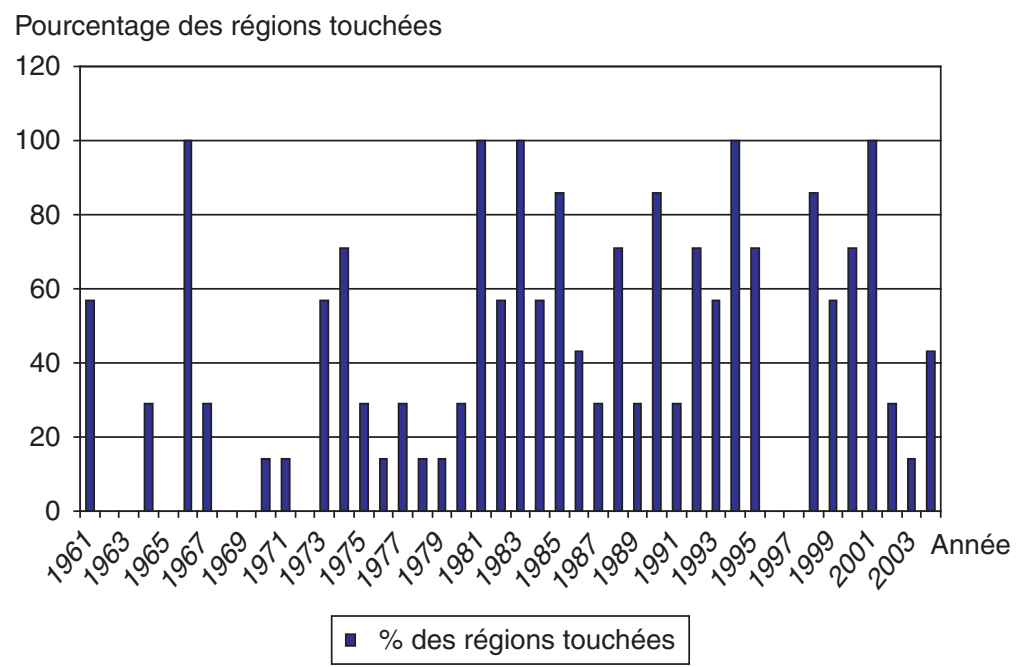

Fig. 10. Pourcentage des régions touchées par les différentes années de sécheresse à l'échelle nationale.

Fig. 10. Percentage of regions touched by drought on a national scale.

- après 1972, on a eu de plus en plus de sécheresses touchant souvent la majorité des villes;

- sur les 44 ans étudiés, 36 ont vu la sécheresse toucher au moins une des sept villes. Cinq sécheresses ont concerné l'ensemble des villes;

- les douze dernières années ont connu dix années de sécheresse partielles dont deux généralisées, ce qui en fait la décennie la plus sèche.

\section{Sévérité de la sécheresse}

En vue de caractériser les sécheresses vécues et leur niveau de sévérité, nous avons calculé pour les différentes villes au cours de la période d'étude l'indice standardisé de précipitations et l'indice de nombre d'écart type (Fig. 11). II en résulte que la fréquence des sécheresses modérées varie entre $20 \%$ à Marrakech et $34 \%$ à Ifrane et Agadir. Celle des sécheresses sévères oscille entre $23 \%$ et $9 \%$ au sein des mêmes villes. La période 1961-2004 n'a pas connu de sécheresses extrêmes (Tab. VIII).

La probabilité d'occurrence d'une année sévèrement sèche varie d'une année sur quatre pour Marrakech à une année sur onze pour Casablanca et Agadir, par contre celle d'une année modérément sèche varie d'une année sur cinq pour Marrakech à une année sur trois pour le reste des villes. Ainsi la probabilité d'occurrence de types de sécheresse diminue avec l'augmentation de sa sévérité (Fig. 11).

Pour mieux appréhender la sécheresse et sa dynamique à l'échelle 

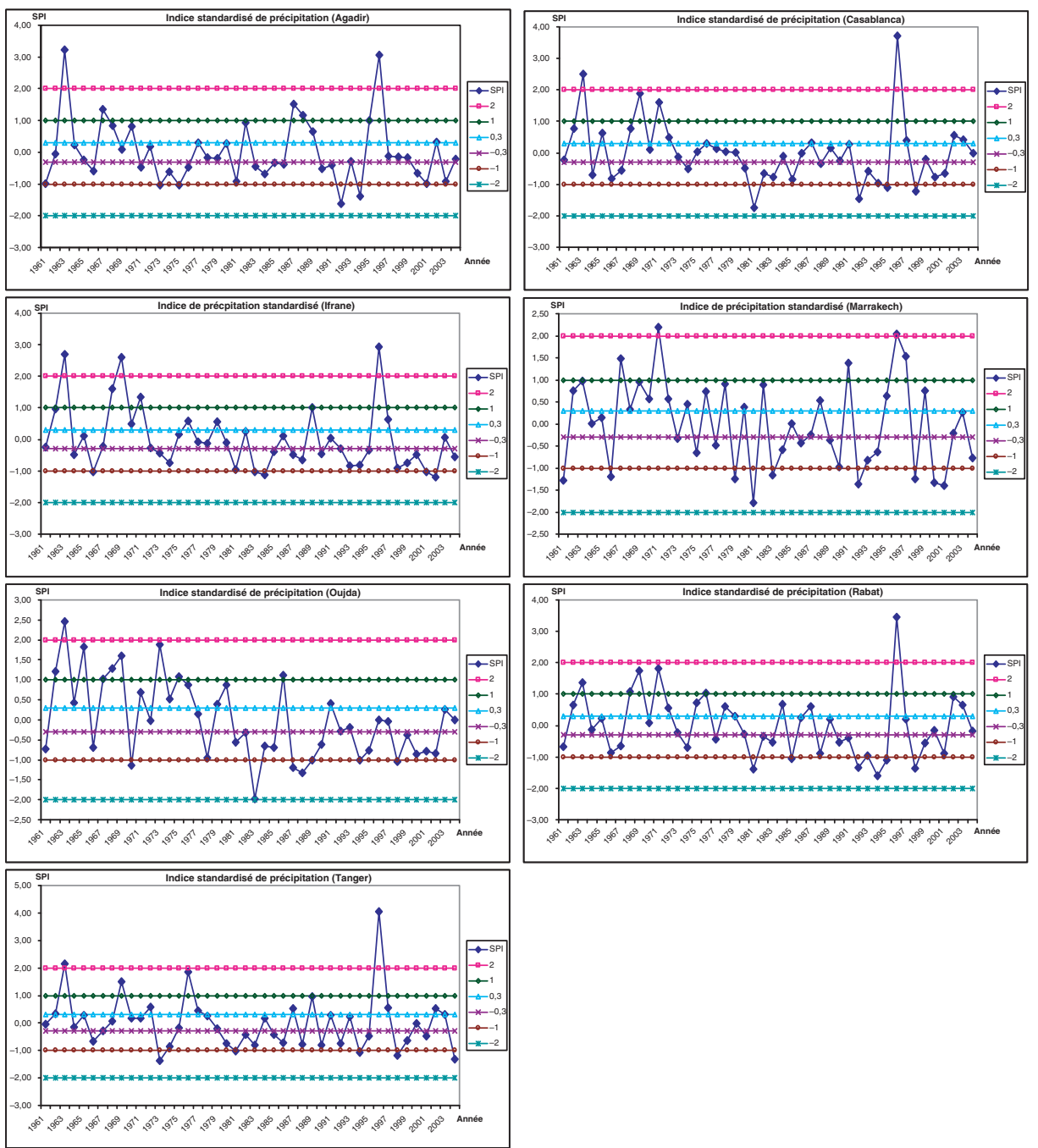

Fig. 11. Évolution de l'indice standardisé de précipitation au cours de la période 1961-2004 dans les sept villes.

Fig. 11. Standardized precipitation index evolution during the period 1961-2004 in the seven cities.

saisonnière, une évaluation de l'indice standardisé de précipitation a été faite à cette échelle. Les résultats présentés sur la figure 12 indiquent que les sécheresses d'automne et de printemps sont les plus fréquentes, suivies de celles d'hiver et de printemps. On se retrouve alors souvent avec des 
Tableau VIII. Fréquence de différentes classes de la sécheresse au cours de la période 1961-2004. Table VIII. Different drought classes frequency during the period 1961-2004.

\begin{tabular}{lccc}
\hline Villes & $\begin{array}{c}\text { Fréquence des } \\
\text { sécheresses modérées } \\
(\%)\end{array}$ & $\begin{array}{c}\text { Fréquence des } \\
\text { sécheresses sévères } \\
(\%)\end{array}$ & $\begin{array}{c}\text { Fréquence des } \\
\text { sécheresses extrêmes } \\
(\%)\end{array}$ \\
\hline Agadir & 34 & 9 & 0 \\
Casablanca & 30 & 9 & 0 \\
Ifrane & 34 & 11 & 0 \\
Marrakech & 20 & 23 & 0 \\
Oujda & 30 & 16 & 0 \\
Rabat & 30 & 14 & 0 \\
Tanger & 30 & 11 & 0 \\
\hline
\end{tabular}

Pourcentage des régions touchées par les sécheresses saisonnières

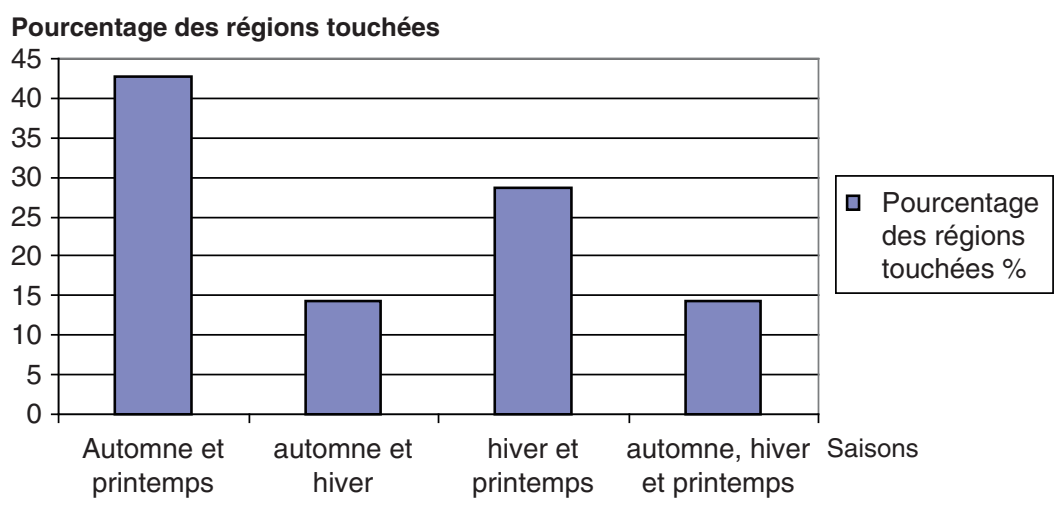

Fig. 12. Analyse des sécheresses saisonnières au niveau des sept villes pour la période 1961-2004. Fig. 12. Seasonal droughts during the period 1961-2004 in the seven cities.

années à deux saisons au lieu de quatre (hiver - été).

\section{CONCLUSION}

Ce travail de recherche a permis de faire ressortir ce qui suit :

- la sécheresse s'est imposée avec force ces dernières décennies en tant qu'élément structurel du climat du Maroc ;

- les précipitations ont connu globalement une tendance à la baisse : l'occurrence d'années humides généralisées sur l'ensemble de territoire devient une exception ;

- un réchauffement du climat significatif accompagne cette installation de la sécheresse au Maroc avec une évapotranspiration correspondante qui croît aussi. II en résulte un déficit hydrique de plus en plus marqué qui fragilise l'équilibre offre-demande;

- à l'échelle saisonnière, on perçoit une diminution de l'importance de printemps et de l'automne. On 
s'oriente vers un climat bi-saison : hiver-été ;

- dans ce comportement de la sécheresse dans notre région, les douze dernières années se sont distinguées et ce en conformité avec ce qui a été noté au niveau planétaire (GIEC, 2007) ;

- l'ensemble des constats faits dans cette recherche conforte à l'échelle nationale les résultats des travaux du quatrième rapport de GIEC (GIEC, 2007) et nous permettent de mieux préciser le poids de la sécheresse dans le climat de notre région. II reste maintenant à s'adapter à ce nouveau contexte climatique et aux sécheresses associées, surtout que les travaux de GIEC (GIEC, 2007) ont indiqué que cette tendance au réchauffement et à l'assèchement devrait continuer;

- la grande question qui reste posée est de savoir comment cette tendance à l'assèchement de la région du Maroc risque d'évoluer durant ce siècle en relation avec les changements climatiques prévus. Dans ce sens, cette recherche a été poursuivie en recourant à des modèles de circulation générale pour prévoir le réchauffement et la dynamique de sécheresse attendus au Maroc en 2050. Les résultats de cette recherche ont été publiés dans la revue marocaine de génie civil (Stour \& Agoumi, 2007).

\section{RÉFÉRENCES BIBLIOGRAPHIQUES}

Aghrab A., 2003. Caractérisation de la sécheresse et élaboration des indicateurs climatiques pour son alerte précoce dans la région de Saïss. Mémoire de fin d'éudes. École Nationale d'Agriculture de Meknès, Maroc.

Agoumi A. \& Debbarh A., 2005. Ressources en eau et bassins versants du Maroc : 50 ans de développement (1955-2005). In 50 ans de développement humain et perspectives 2025 : Cadre naturel, environnement et territoires. Éditeurs : A. Agoumi et al., pp. 9-58.

Barakat F. \& Handoufe A., 1998. Approche agroclimatique de la sécheresse agricole au Maroc. Sécheresse $9: 201-208$.

Bootsma A., Boisvert J.B., De Jong R. \& Baier W., 1996. La sécheresse et l'agriculture canadienne : Une revue des moyes d'action. Sécheresse 7 : 277-285.

Direction de la Météorologie Nationale, 1995. Spécial sécheresse. Bulletin d'information $d u$ Centre National $d u$ Climat et des Recherches Météorologiques, $\mathrm{N}^{\circ} 2$.

GIEC, 2007. Bilan 2007 des changements climatiques : Les bases scientifiques physiques. In Quatrième rapport d'évaluation de GIEC. 2 février 2007, France.

Hayes M.J., Svoboda M.D., Wilhite D.A., \& Vanyarkho O.V., 1999. Monitoring the 1996 Drought using the Standardized Precipitation Index. Bulletin of the American Météorologic Society 3 : 429-438.

Mckee T.B., Doesken N.J. \& Kleist J., 1993. The relationship of drought frequency and duration times scales. American Meteorological Society. 8th conference on Applied Climatology, 17-22 Janvier, Anaheim, CA, pp.179-184.

Mckee T.B., Doesken N.J. \& Kleist J., 1995. Drought monitoring with multiple times scales. American Meteorological Society. 9th conference on Applied Climatology, 15-22 Janvier, Dallas, TX, pp. 233-236. 
Ministère de l'équipement, de l'agriculture et de l'environnement, 1997. Un siècle d'observations météorologiques : Sécheresse et gestion de l'eau au Maroc. Rabat : Publications du département de l'équipement, 3/1997. 119p.

Rognon P., 1997. Sécheresse et Aridité : Leur impact sur la désertification au Maghreb. Sécheresse, 7 : 287-297.

Safi H., 1990. Essai sur l'économie de la sécheresse au Maroc 1493-1986, passé présent et perspectives. Thèse université Mohamed V, Rabat : 190 p.

Stockton C.W., 1988. Current research progress toward understanding drought. In proceed. Confer. On drought, water management an food production, 21-24 novembre 1988. Agadir Maroc : 21-35.
Stour L. \& Agoumi A., 2007. Réchauffement climatique et sécheresse au Maroc en 2050. Revue Marocaine de Génie Civil (119), $3^{\mathrm{e}}$ trimestre 2007.

Tilioua A. \& Mechouary R., 2006. Maroc et changements climatiques. Mémoire de fin d'études. EHTP. Maroc.

Wilhite D.A. \& Svoboda M.D., 2000. In : Proceeding of the meeting : Improving Drought Early Warning Systems in the Context of Drought Preparedness and Mitigation, 5-7 Septembre 2000. Lisbon, Portugal 1-21.

Yacoubi M., El Mourid M., Chbouki N. \& Stockle C., 1998. Typologie de la sécheresse et recherche d'indicateur d'alerte en climat semi-aride marocain. Sécheresse 9 : 269-276. 